\title{
Three-particle quantization condition: an update
}

\section{Maxwell T. Hansen*}

Physics Department, University of Washington, Seattle, WA 98195-1560, USA

E-mail: mth28@uw. edu

\section{Stephen R. Sharpe}

Physics Department, University of Washington, Seattle, WA 98195-1560, USA

E-mail: srsharpeduw. edu

We give an update on our derivation of a quantization condition relating the finite-volume spectrum of three particles in a cubic box to infinite-volume scattering quantities. We have discovered and fixed technical problems in the derivation sketched in our proceedings from last year's lattice conference, and have presented a detailed description of the corrected derivation in a recent paper. Here we give an overview of the problems and their solutions, and describe open questions.

The 32nd International Symposium on Lattice Field Theory,

23-28 June, 2014

Columbia University New York, NY

* Present address: Institut für Kernphysik and Helmholz Institute Mainz, Johannes Gutenberg-Universität Mainz, 55099 Mainz, Germany

† Speaker. 


\section{Introduction}

In this talk we give an update on our derivation of a quantization condition relating the finitevolume (FV) spectrum in the three-particle sector to infinite-volume scattering quantities. At last year's lattice conference we presented a preliminary result [1]. This, however, turned out not to be fully correct, due to technical issues in the derivation. We have recently finished a corrected derivation, written up at length in Ref. [2]. We aim to give here an overview of the result and a sketch of the technical issues that have arisen.

Many resonances have significant coupling to three-particle channels, e.g. $\omega \rightarrow 3 \pi, K^{*} \rightarrow$ $K \pi \pi$ and $N(1440) \rightarrow N \pi \pi$, and if we wish to determine the properties of such resonances from first principles using lattice QCD, a formalism connecting the FV spectrum to infinite-volume (measurable) quantities is needed. ${ }^{1}$ Additionally, three-body interactions are important in nuclear physics and perhaps also in pion- and kaon-condensed matter. A formalism is needed to extract these interactions from the FV spectrum. Finally, interesting weak decays involve three particles, e.g. $K \rightarrow 3 \pi$, and in order to study these one needs as a first step to understand the FV effects on the three-particle system.

The three-particle quantization condition is at the frontier of finite-volume formalism. The two-particle quantization condition is well understood. This formalism was first developed by Lüscher [4, 5], and is now widely implemented in numerical calculations. By contrast, although important progress has been made $[6,7]$, our current understanding of the three-particle sector is incomplete.

The work reviewed here is applicable to any relativistic field theory with a single scalar field (of mass $m$ ), with the only simplification being that we impose a $Z_{2}$ symmetry (akin to G-parity) such that all vertices have an even number of legs. This means that the relevant physical scattering amplitudes are those for $2 \rightarrow 2$ and $3 \rightarrow 3$ processes, while $2 \rightarrow 3$ transitions do not occur. We work to all orders in perturbation theory, and do not assume that couplings are weak. On the FV side we assume the simplest possible implementation: a periodic cubic box with side length $L$. We expect that extensions to non-identical particles, to spin, and to asymmetric boxes will be relatively straightforward using methods developed for two particles.

\section{Quantization conditions}

It is useful to recall the two-particle single-channel quantization condition in a form similar to that derived in Ref. [8]. For fixed total momentum $\vec{P}, \mathrm{FV}$ states have energies, $E$, such that ${ }^{2}$

$$
\operatorname{det}\left(F_{2}^{-1}+i \mathscr{K}_{2}\right)=0 .
$$

Here $\mathscr{K}_{2}$ is the two-particle $\mathrm{K}$-matrix, an infinite-volume quantity, while $F_{2}$ is a known kinematical factor, dependent on the volume. Both quantities are matrices in the space of angular momentum in the two-particle center-of-mass $(\mathrm{CM})$ frame, e.g. $\left(F_{2}\right)_{\ell_{1}, m_{1} ; \ell_{2}, m_{2}}$. This space arises because, for given $(E, \vec{P})$, the only degree of freedom left for two on-shell particles is the direction of momentum

\footnotetext{
${ }^{1}$ Indeed, the recent coupled-channel analysis of Ref. [3] is limited at the highest energies by the lack of a threeparticle formalism, a problem that will become more acute as quark masses are lowered towards their physical values.

${ }^{2}$ This result holds up to exponentially suppressed FV effects $\left(\propto e^{-m L}\right)$ which we ignore throughout.
} 
for one of the particles in the CM frame. By decomposing in spherical harmonics, this degree of freedom may be equivalently expressed as the $\mathrm{CM}$ angular momentum. While $\mathscr{K}_{2}$ is diagonal in angular momentum, $F_{2}$ knows about the box shape and mixes angular momenta. Roughly speaking, $F_{2}$ is the difference between the FV momentum sum and infinite-volume integral over a two-particle cut. It is expressible in terms of Lüscher zeta functions [4]. The beauty of Eq. (2.1) is that it separates finite and infinite-volume quantities. ${ }^{3}$

The extension to three particles requires additional kinematical variables. The choice we find convenient divides the particles (arbitrarily) into a "spectator" and the "scattering pair". The variables are then the FV momentum of the spectator, $\vec{k}=2 \pi \vec{n} / L$, and the angular momentum of the scattering pair in their CM frame. Thus each matrix "index" is written $\vec{k}, \ell, m$. With this change, our result for the three-particle quantization condition can be written in a form that is superficially similar to the two-particle result (2.1), namely

$$
\operatorname{det}\left(F_{3}^{-1}+i \mathscr{K}_{\mathrm{df}, 3}\right)=0 .
$$

However, $F_{3}$ is not a purely kinematical function as it depends on $\mathscr{K}_{2}$ :

$$
F_{3}=\frac{1}{2 \omega L^{3}}\left[-\frac{2 F}{3}+\frac{1}{F^{-1}+(1+\mathscr{K} G)^{-1} \mathscr{K} F}\right] \text {. }
$$

Here all quantities are matrices in the enlarged space. $\mathscr{K}$ and $F$ are simply $\mathscr{K}_{2}$ and $F_{2}$, respectively, multiplied by $\delta_{\vec{k}, \vec{k}^{\prime}}$, the prefactor $\omega L^{3}$ is a diagonal matrix with entries $\sqrt{\vec{k}^{2}+m^{2}} L^{3}$, and $G$ is a regulated free non-relativistic propagator (defined precisely in Ref. [2]). In principle, $\mathscr{K}$ can be determined by interpolating results obtained from the two-particle quantization condition, in which case $F_{3}$ is known and one can use Eq. (2.2) to determine $\mathscr{K}_{\mathrm{df}, 3}$.

The forms of Eqs. (2.2) and (2.3) are (aside from minor changes in notation) identical to those given in last year's proceedings [Eqs. (6.1) and (6.2) of Ref. [1]]. There have, however, been two very important changes in the meaning of the symbols. The first concerns $F$, which is a sum-integral difference. The integral part is defined using a modified principal value prescription (denoted $\widetilde{P V}$ ) which was incompletely specified in Ref. [1], but is completely defined in Ref. [2].

The second change concerns $\mathscr{K}_{\mathrm{df}, 3}$ and is more significant. The good news is that this an infinite-volume three-to-three scattering quantity, so that the FV spectrum is determined using Eq. (2.2) in terms of infinite-volume quantities. The bad news is that, contrary to our claim last year, the relation of $\mathscr{K}_{\mathrm{df}, 3}$ to the three-particle scattering amplitude is not yet clear. We strongly suspect, however, that such a relation exists, for it does in the non-relativistic limit [6]. $\mathscr{K}_{\mathrm{df}, 3}$ is defined in Ref. [2] as a sum of the same diagrams which build up the divergence-free three-to-three scattering amplitude, ${ }^{4}$ except that the $\widetilde{\mathrm{PV}}$ prescription is used, which requires specification of the ordering of integrations, and that additional "decorations" must be added to the diagrams.

In the following two sections we give some further details concerning these two changes.

\footnotetext{
${ }^{3}$ The result of Ref. [8] is actually $\operatorname{det}\left(F_{i \varepsilon}^{-1}+i \mathscr{M}\right)=0$, with $\mathscr{M}$ the scattering amplitude and $F_{i \varepsilon}$ a modified kinematical factor. This form is equivalent to Eq. (2.1), as shown in Ref. [2], and the latter is more useful for our purposes.

4 "Divergence-free" indicates that divergence in the physical three-to-three scattering amplitude (which occurs for on-shell above-threshold momenta) is subtracted. For details see Refs. [1,2]. This aspect of the problem was understood last year, and we do not discuss it further.
} 


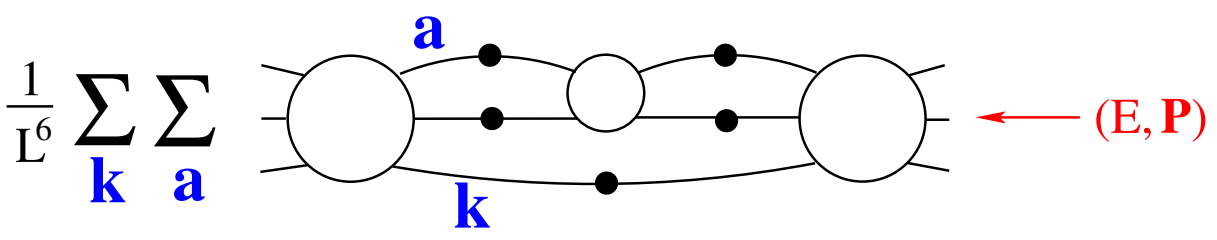

Figure 1: Segment of a Feynman diagram contributing to FV correlator. Open circles and lines are BS kernels and fully-dressed propagators, respectively. See text for further discussion.

\section{Cusps and their removal with the $\widetilde{\mathrm{PV}}$ prescription}

Our analysis considers a FV correlation function in the sector with an odd number of particles, evaluated in Minkowski space as a function of $E$ (and for fixed $\vec{P}$ and $L$ ). Poles in $E$ then give the FV spectrum. Power-law FV effects in the correlator arise from the differences between loop sums and integrals over singular (but integrable) summands. Such singularities occur only when there are three-particle intermediate states. An example is the "cut" which runs through the momenta $\vec{a}$ and $\vec{k}$ in the diagram of Fig. 1. Our analysis consists of replacing the FV momentum sums with infinitevolume integrals plus a residue that brings in the factors of $F$. The need for the $\widetilde{\mathrm{PV}}$ prescription arises from cuts where one or both sides contains a two-particle Bethe-Salpeter (BS) kernel (so that there is a spectator). This is true for either cut in Fig. 1, and we focus here on the cut on the left side.

Sums can be replaced by integrals (up to exponentially suppressed corrections) for non-singular summands. Thus we need consider only the singular part of the summand, which can be written

$$
\frac{1}{L^{6}} \sum_{\vec{k}} \sum_{\vec{a}} \frac{A(\vec{k}, \vec{a}) B(\vec{k}, \vec{a})}{E-\omega_{k}-\omega_{a}-\omega_{k a}} .
$$

Here the sums run over FV momenta, $A$ and $B$ are smooth functions representing the kernels respectively to the left and right of the cut, and $\omega_{k}, \omega_{a}$ and $\omega_{k a}$ are the on-shell energies of the three cut particles [e.g. $\omega_{k a}=\sqrt{(\vec{P}-\vec{k}-\vec{a})^{2}+m^{2}}$ ].

The first step is to make the replacement

$$
L^{-3} \sum_{\vec{a}}=(2 \pi)^{-3} \int d^{3} \vec{a}+\left[L^{-3} \sum_{\vec{a}}-(2 \pi)^{-3} \int d^{3} \vec{a}\right] .
$$

The sum-integral difference leads to the kinematical factor $F$ multiplied by the on-shell values of $A$ and $B$ [8]. To define $F$ we need to specify a pole prescription for the integral, and, for reasons to be seen shortly, we use $\widetilde{\mathrm{PV}}$ instead of the $i \varepsilon$ prescription used in Ref. [8]. For the $F$ term the remaining sum over $\vec{k}$ must remain a sum, since $F$ has singularities as a function of $\vec{k}$. This sum over $\vec{k}$, together with the dependence of $F$ on the two-particle angular momentum $\ell, m$, builds up the matrix indices introduced above.

The second step is to deal with the $\vec{a}$ integral in (3.2). This is acted on by a sum over $\vec{k}$, and we would like to replace this outer sum with an integral. While the integral over $\vec{a}$ does lead (for $i \varepsilon, \mathrm{PV}$ and $\widetilde{\mathrm{PV}}$ pole prescriptions) to a finite function of $\vec{k}$, it does not in general yield a smooth 

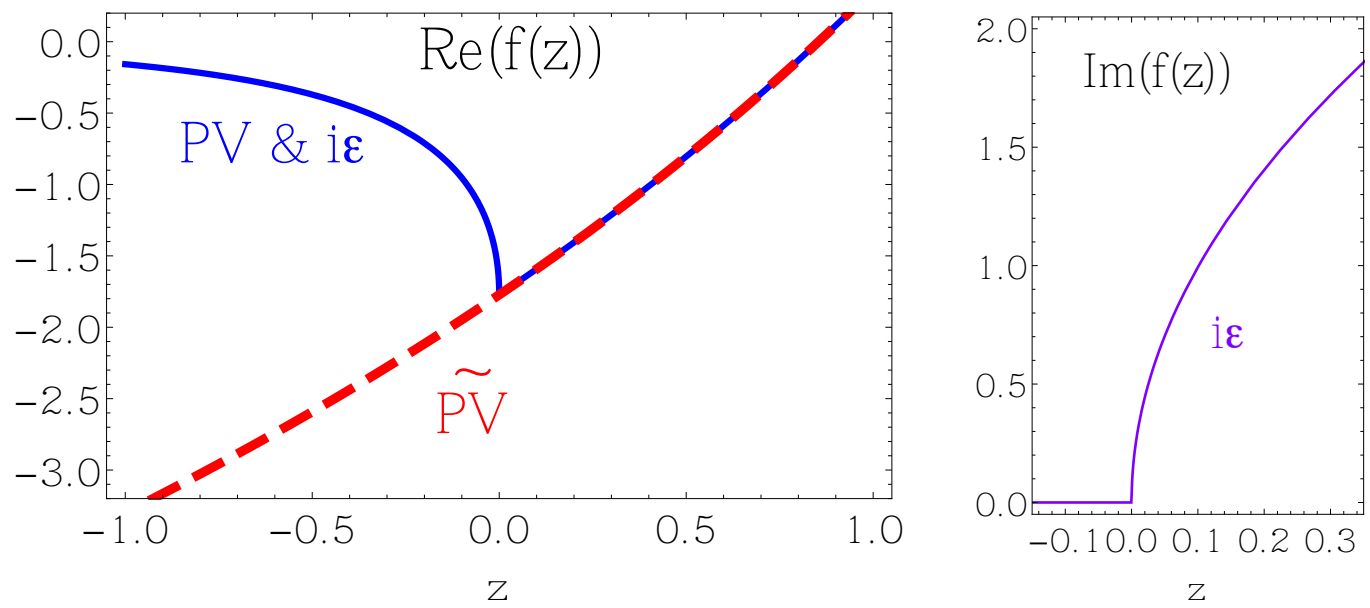

Figure 2: Results for the integral of Eq. (3.3).

function. In particular, there can be cusps when $\vec{k}$ is such that the scattering pair is at threshold. ${ }^{5}$ If cusps are present then the difference between the sum and integral over $\vec{k}$ gives rise to power-law dependence on $L$ (beginning at $1 / L^{4}$ ) that one must explicitly include to derive the quantization condition. Our choice is to use a pole prescription which is cusp free, namely the $\widetilde{\mathrm{PV}}$ prescription. Then we can simply replace the sum over $\vec{k}$ with an integral. We stress that this issue arises first for three particles because only then do we need to consider the analytic properties of the sum-integral difference below the two-particle threshold.

The presence of cusps is well known using the standard ic prescription, and is indeed required by unitarity. To illustrate the cusps that we encounter in the present context and to better explain the $\widetilde{P V}$ prescription we consider a simple integral that (as explained in App. B of Ref. [2]) captures the essential features of the actual integral:

$$
f(z)=\int_{0}^{\infty} d x \sqrt{x} \frac{e^{z-x}}{z-x} .
$$

Results for $f(z)$ using the $i \varepsilon$, standard PV, and $\widetilde{\mathrm{PV}}$ prescriptions are shown in Fig. 2. Observe that both $i \varepsilon$ and PV prescriptions lead to cusps at $z=0$ (which corresponds to the two-particle threshold in this example). For the $i \varepsilon$ prescription cusps appear in both real and imaginary parts. For the PV prescription, which is equivalent to the real part of the $i \varepsilon$ prescription, we have only the real cusp. As we show in Ref. [2], and is likely well known in the three-body community (see, e.g., Ref. [6]), the difference between the cusped PV result and the smooth extension of the $z>0$ curve (i.e. the red dashed curve in Fig. 2) can be calculated analytically. For the integral of actual interest, the difference depends on the analytic continuation of the product of on-shell amplitudes multiplied by an analytically continued phase-space factor. The $\widetilde{\mathrm{PV}}$ prescription consists in subtracting this difference, and then smoothly turning off the subtraction far below threshold. Slightly below threshold, this yields the (unique) analytic continuation of the above-threshold standard PV result.

\footnotetext{
${ }^{5}$ Note that when $|\vec{k}|$ is large enough, the scattering pair lies below threshold.
} 


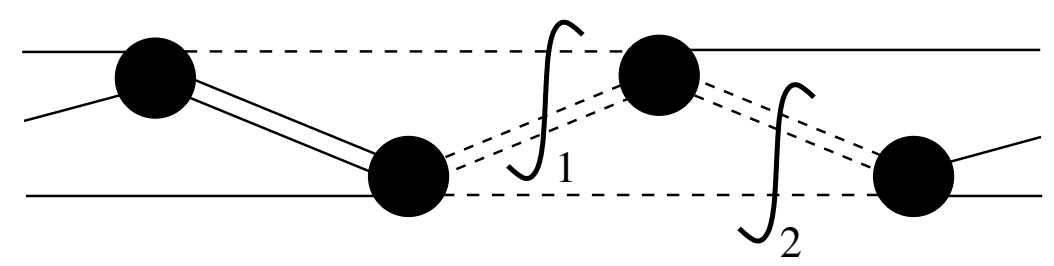

Figure 3: Example of a two-loop contribution to $\mathscr{K}_{\mathrm{df}, 3}$. Notation as follows: filled circles: $\mathscr{K}_{2}$; double dashed lines: divergent part of propagator; double solid lines: finite part of propagator; single dashed lines: on-shell particle; single solid lines: amputated on-shell external propagator. See Ref. [2] for more details.

Using the $\widetilde{\mathrm{PV}}$ prescription throughout, we are able to separate FV and infinite-volume contributions. In the two-particle subsector (e.g. when iterating insertions of the two-to-two BS kernel in Fig. 1) this leads to the appearance of a two-particle K-matrix, $\mathscr{K}_{2}$, rather than the corresponding scattering amplitude. $\mathscr{K}_{2}$ is the standard K-matrix above threshold (where PV and $\widetilde{\mathrm{PV}}$ are equivalent), but just below threshold corresponds to the analytic continuation of the above-threshold function. For a given value of $\ell$, it is simply $16 \pi E^{*} /\left(a^{*} \cot \delta_{\ell}\right)$ above threshold (with $E^{*}$ and $a^{*}$ respectively the two-particle $\mathrm{CM}$ energy and momentum), while the result below threshold is defined via the standard threshold expansion. This is, in fact, the standard choice in the literature when using the two-particle quantization condition below threshold to study FV effects on bound states. In this sense, our prescription is not new (aside from the turn-off of the subtraction far below threshold).

\section{Enforcing particle-interchange symmetry}

While the $\widetilde{\mathrm{PV}}$ prescription avoids the issue of cusps, it introduces substantial complications into the definition of $\mathscr{K}_{\mathrm{df}, 3}$. This is very difficult to explain without going into technicalities, so here we give only a crude sketch of the issues. For the full story see Ref. [2].

The basic issue is that the $\widetilde{\mathrm{PV}}$ prescription breaks particle-interchange symmetry. One of the particles (the spectator) is treated differently from the other two. One consequence is that in diagrams containing adjacent two-to-two BS kernels on different pairs (i.e. where the spectator changes) the order of $\widetilde{\mathrm{PV}}$-regulated integrals matters. This is not the case with the $i \varepsilon$ prescription. An example is shown in Fig. 3, which is one of many two-loop contributions to $\mathscr{K}_{\mathrm{df}, 3}$. Here we have performed a summation of two-to-two BS kernels into K-matrices. Our construction requires that the two $\widetilde{P V}$-regulated integrals be done in the order shown. One also sees here another complication: defining a divergence-free three-particle scattering quantity requires that the central propagators are broken up into singular and finite parts. Our construction gives a prescription for this breakup, and the resulting order of integrals, for diagrams of all orders.

A second consequence of the breaking of particle-interchange symmetry by the $\widetilde{P V}$ prescription is that the resulting infinite-volume quantity, $\mathscr{K}_{\mathrm{df}, 3}$, is not manifestly symmetric. More precisely, while it is symmetric under interchange of external legs, it contains internally some parts that are asymmetric. We refer the reader to Ref. [2] for complete details. Again, a precise formula for these asymmetric parts can be given for all diagrams. 
Because of the complicated definition of $\mathscr{K}_{\mathrm{df}, 3}$, we have, so far, been unable to determine its relation to the physical three-to-three scattering amplitude. We have evidence that such a relation exists close to threshold from the work of Ref. [6], and thus we are presently concentrating on the threshold expansion to address this question.

\section{Outlook}

Many issues have not been discussed in this short presentation. For example, how can the results (2.2) and (2.3) be checked? A major check is the agreement of our results near the threeparticle threshold to those obtained from non-relativistic quantum mechanics. This was mentioned last year [1] and we are presently writing up a detailed discussion. Are these complicated formulae useful in practice? This we have begun to address in Ref. [2]. As in the two-particle case, practical utility requires truncation of the infinite-dimensional matrices to a finite subspace. We have shown in Ref. [2] that this can be done in principle, and have worked out in detail the simplest truncation. More work is needed here, for example using model amplitudes to illustrate in detail how the result may be applied.

In closing, we stress again that the most important unresolved issue is to determine the relation of $\mathscr{K}_{\mathrm{df}, 3}$ to physical scattering amplitudes.

\section{Acknowledgments}

We thank Raúl Briceño, Zohreh Davoudi and Akaki Rusetsky for helpful discussions. This work was supported in part by the United States Department of Energy grants DE-FG02-96ER40956 and DE-SC0011637. MTH was supported in part by the Fermilab Fellowship in Theoretical Physics. Fermilab is operated by Fermi Research Alliance, LLC, under Contract No. DE-AC0207CH11359 with the United States Department of Energy.

\section{References}

[1] M. T. Hansen and S. R. Sharpe, PoS LATTICE2013, 221 (2013), arXiv:1311.4848 [hep-lat] .

[2] M. T. Hansen and S. R. Sharpe, Phys.Rev. D90, 116003 (2014), arXiv:1408.5933 [hep-lat] .

[3] J. J. Dudek et al. (Hadron Spectrum), Phys.Rev.Lett. 113, 182001 (2014), arXiv:1406.4158 [hep-ph] .

[4] M. Luscher, Commun.Math.Phys. 105, 153 (1986).

[5] M. Luscher, Nucl.Phys. B354, 531 (1991).

[6] K. Polejaeva and A. Rusetsky, Eur.Phys.J. A48, 67 (2012), arXiv:1203.1241 [hep-lat] .

[7] R. A. Briceno and Z. Davoudi, Phys.Rev. D87, 094507 (2013), arXiv:1212.3398 [hep-lat] .

[8] C. Kim, C. Sachrajda, and S. Sharpe, Nucl.Phys. B727, 218 (2005), arXiv:hep-lat/0507006 . 